\title{
Nailing Natural SUSY: Higgsino Parameter Determination at the ILC
}

\section{Mikael Berggren ${ }^{a}$, Felix Brümmer ${ }^{a}$, Jenny List ${ }^{a}$, Gudrid Moortgat-Pick ${ }^{b}$, Tania Robens $^{c}$, Krzysztof Rolbiecki ${ }^{d}$ and Hale Sert ${ }^{* a b}$}

${ }^{a}$ DESY, Notkestraße 85, 22607 Hamburg, Germany

${ }^{b}$ University of Hamburg, Inst. f. Experimantal Physics and II. Inst. f. Theoretical Physics

Luruper Chaussee 149, 22761 Hamburg, Germany

'IKTP, TU Dresden, Zellescher Weg 19, 01069 Dresden, Germany

dIFT-UAM/CSIC, Nicolás Cabrera 13-15, 28049 Madrid, Spain

E-mail: mikael.berggren@desy.de, felix.bruemmeredesy.de,

jenny.list@desy.de, gudrid.moortgat-pick@desy.de,

tania.robens@desy.de, krzysztof .rolbiecki@desy.de,

hale.sertedesy.de

In unconstrained SUSY models, the most natural value for the $\mu$ parameter is not far from the $Z^{0}$ mass, predicting the lightest gauginos to be higgsino-like and nearly degenerate in mass, with mass splittings of a few $\mathrm{GeV}$ or even of less than a GeV. At the ILC, $\tilde{\chi}_{1}^{0} \tilde{\chi}_{2}^{0}$ and $\tilde{\chi}_{1}^{+} \tilde{\chi}_{1}^{-}$production can be detected via the recoil against hard photon radiation and even sub-GeV mass differences can be reconstructed from the kinematics of the very soft visible decay products. The expected precisions on the polarised cross sections, on the $\tilde{\chi}_{1}^{ \pm}$and $\tilde{\chi}_{2}^{0}$ masses and on their mass differences to the $\tilde{\chi}_{1}^{0}$ have been investigated with the ILD detector concept at the centre-of-mass energies of 350 and $500 \mathrm{GeV}$, taking into account realistic assumptions on the beam parameters and beaminduced backgrounds. From these observables alone, the $\mu$ parameter can be determined to the level of a few percent, while $M_{1}$ and $M_{2}$ can be constrained within 10-20\% even if they are in the multi- $\mathrm{TeV}$ regime.

The European Physical Society Conference on High Energy Physics -EPS-HEP2013

18-24 July 2013

Stockholm, Sweden

\footnotetext{
*Speaker.
} 


\section{Introduction}

The Minimal Supersymmetric Standard Model (MSSM) is a well-known extension of the Standard Model (SM), which can solve most of the SM problems, such as dark matter, grand unification and hierarchy. The higgsino mass parameter $\mu$ is the only dimensionful parameter in the MSSM and the only mass parameter allowed by unbroken SUSY. Its value is expected to be near the electroweak scale for naturalness reasons. This motivates different scenarios which can contain light higgsino-like charginos and neutralinos at the electroweak scale and no other supersymmetric particles below the $\mathrm{TeV}$ scale. In this study, a scenario containing three almost mass degenerate higgsinos, $\tilde{\chi}_{1}^{ \pm}, \tilde{\chi}_{1}^{0}$ and $\tilde{\chi}_{2}^{0}$ is studied at the International Linear Collider (ILC) which is an electron-positron collider planned to complement the LHC. The higgsinos can be detected via two production processes, $e^{+} e^{-} \rightarrow \tilde{\chi}_{1}^{+} \tilde{\chi}_{1}^{-}$and $e^{+} e^{-} \rightarrow \tilde{\chi}_{1}^{0} \tilde{\chi}_{2}^{0}$, where $\tilde{\chi}_{1}^{ \pm} \rightarrow \tilde{\chi}_{1}^{0} W^{ \pm *}$ and $\tilde{\chi}_{2}^{0} \rightarrow \tilde{\chi}_{1}^{0} Z^{0 *}$ or $\tilde{\chi}_{2}^{0} \rightarrow \tilde{\chi}_{1}^{0} \gamma$. Since the mass difference of the $\tilde{\chi}_{1}^{ \pm}$and $\tilde{\chi}_{2}^{0}$ with $\tilde{\chi}_{1}^{0}$ (LSP) is less than a few GeV, there are only a few soft visible particles and lots of missing energy due to the two LSPs in the final state. To observe this kind of final states at the LHC is challenging; however, it is possible at the ILC. The analysis is based on fast simulation of the ILD detector concept [1] as implemented in SGV [2]. Detailed information about the analysis can be found in reference [3].

\section{Analysis}

In this study, a hard initial state radiation photon is required to get rid of the similarity of the final state of the signal with some SM backgrounds, in particular two photon processes $e^{+} e^{-} \rightarrow e^{+} e^{-} \gamma \gamma \rightarrow e^{+} e^{-} f \bar{f}$. The analysis is performed at $500 \mathrm{GeV}$ center of mass energy assuming $500 \mathrm{fb}^{-1}$ integrated luminosity for each combination of beam polarisations, $P\left(e^{+}, e^{-}\right)=$ $( \pm 30 \%, \mp 80 \%)$. The input masses of the $\tilde{\chi}_{1}^{ \pm}, \tilde{\chi}_{1}^{0}$ and $\tilde{\chi}_{2}^{0}$ are $167.36 \mathrm{GeV}, 166.59 \mathrm{GeV}$ and 167.63 $\mathrm{GeV}$, respectively.

Event Selection: First of all, a pre-selection is applied to suppress the SM background. After that exclusive decay modes are chosen to distinguish the signal processes. For the chargino case, the semileptonic final state with one $\tilde{\chi}_{1}^{ \pm} \rightarrow \pi^{ \pm} \tilde{\chi}_{1}^{0}$ and the other $\tilde{\chi}_{1}^{ \pm} \rightarrow v e^{ \pm}\left(\mu^{ \pm}\right) \tilde{\chi}_{1}^{0}$ is used in the analysis. It occurs in about $35 \%$ of the chargino events. For the neutralino case, the photonic decay mode with a branching ratio of $74 \%$ is selected.

Mass Measurement: The requirement of a hard ISR photon makes it possible to use the reduced center of mass energy $s^{\prime}=s-2 \sqrt{s} E^{\gamma}$ for the higgsino mass measurement. The minimum value for $\sqrt{s^{\prime}}$ is $2 \times M_{\tilde{\chi}_{1}^{ \pm}}$, when both $\tilde{\chi}_{1}^{ \pm}$are produced at rest. Figure 1 (left) shows the $\sqrt{s^{\prime}}$ distribution after the chargino selection as an example. To obtain the higgsino masses, first standard model and then both standard model and signal are fitted with appropriate functions. After fitting, the masses of the higgsinos are obtained as $M_{\tilde{\chi}_{1}^{ \pm}}^{f i t}=168.6 \pm 1.0 \mathrm{GeV}$ and $M_{\tilde{\chi}_{2}^{0}}^{f i t}=166.3 \pm 0.8 \mathrm{GeV}$. Since the method is an approximation, for instance with respect to beam effects, the fitted higgsino masses have been calibrated using Monte Carlo samples with different masses. This results finally in $M_{\tilde{\chi}_{1}^{ \pm}}^{c a l}=167.3 \pm 1.5 \mathrm{GeV}$ and $M_{\tilde{\chi}_{2}^{0}}^{c a l}=165.7 \pm 1.6 \mathrm{GeV}$.

Mass Difference Measurement: For events with $\sqrt{s^{\prime}}$ near its minimal value, the energy of the visible decay products of the $\tilde{\chi}_{1}^{ \pm}$in the chargino rest frame is equivalent to the mass difference. Figure 1 (middle) shows the energy distribution of these decay products in the chargino rest frame 

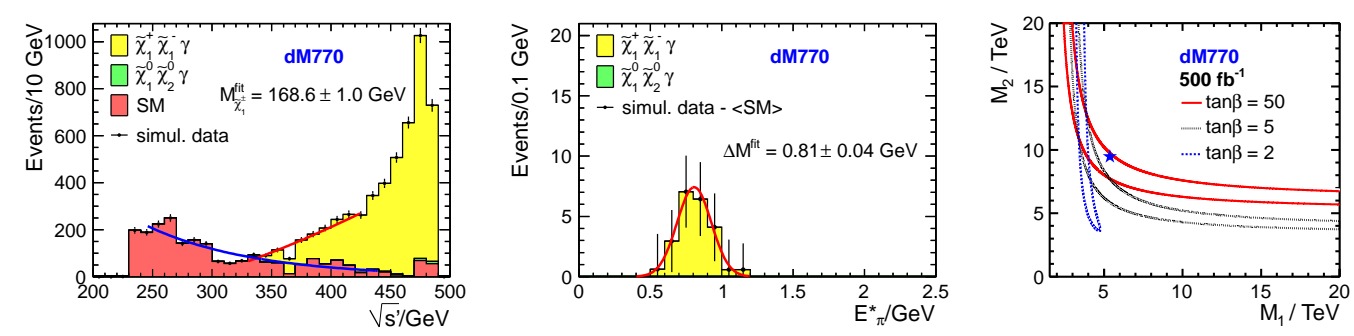

Figure 1: Left: Reduced center of mass energy distribution after the chargino selection, Middle: Energy distribution of visible decay products in the chargino rest frame, Right: The $\Delta \chi^{2}=1$ contours for different $\tan \beta$ values in the $M_{1}-M_{2}$ plane.

after a cut applied on $\sqrt{s^{\prime}}<345 \mathrm{GeV}$ which is close to its minimal value. As seen from the result of the fit, the mass difference can be obtained with $40 \mathrm{MeV}$ precision.

Statistical Precision on Polarised Cross Sections: The expected precisions of the cross section measurements have been estimated by making use of the efficiency and purity. While the efficiencies are not dependent on the polarisation, purity and so precision on the cross section can have a strong dependency on polarisation. We have found that the cross sections can be measured with $1.6 \%(1.7 \%)$ and $3.8 \%(1.9 \%)$ precision for the chargino (neutralino) process for polarisations $P\left(e^{+}, e^{-}\right)=(+30 \%,-80 \%)$ and $P\left(e^{+}, e^{-}\right)=(-30 \%,+80 \%)$, respectively.

Parameter Determination: The results obtained in the previous part of the study are used to determine the mass parameters $M_{1}, M_{2}$ and $\mu$. We have observed that the achieved precision is sufficient to determine the $\mu$ parameter to $\pm 2.5 \mathrm{GeV}$ and to constrain $M_{1}$ and $M_{2}$ to a narrow band in the multi- $\mathrm{TeV}$ regime as shown in Figure 1 (right).

\section{Conclusions}

Light higgsinos are well motivated by naturalness and could be observed at the ILC if they exist. They can be resolved in spite of their small mass differences. Measurement of the higgsino masses and cross sections can be done with statistical precisions sufficient to constrain $M_{1}, M_{2}$ and $\mu$. Moreover, we have observed that the results can be improved with more luminosity and by adding the neutralino mass difference measurement, $\Delta M\left(\tilde{\chi}_{2}^{0}, \tilde{\chi}_{1}^{0}\right)$.

\section{References}

[1] T. Behnke et al., The ILC Technical Design Report - Volume 4: Detectors arXiv:1306.6329 [physics.ins-det].

[2] M. Berggren, $S G V$ 3.0 - a fast detector simulation, arXiv:1203.0217 [physics.ins-det].

[3] M. Berggren, F. Brümmer, J. List, G. Moortgat-Pick, T. Robens, K. Rolbiecki and H. Sert, Tackling light higgsinos at the ILC, arXiv:1307.3566 [hep-ph]. 